\title{
Interscalene brachial plexus blocks in the management of shoulder dislocations
}

\author{
T. J. UNDERHILL, A. WAN \& M. MORRICE \\ Accident and Emergency Department, Derbyshire Royal Hospital, Derby, England
}

\section{SUMMARY}

Interscalene brachial plexus block is a simple and effective alternative to intravenous benzodiazepines or general anaesthesia for manipulation of the dislocated shoulder. Thirty interscalene brachial plexus blocks were performed on 29 patients with dislocations of the shoulder to provide regional anaesthesia for reduction. Pain was abolished by 14 out of the 30 blocks performed, improved by 13 and unchanged by three. Muscle relaxation (MRC grade 3 or less) occurred in 21 patients. In 26 cases the block allowed reduction of the dislocation without additional analgesia or sedative. Reduction was not possible in four cases. There were no significant complications.

\section{INTRODUCTION}

Interscalene brachial plexus block was first described by Winnie in 1970 and has been used in surgery of the shoulder and arm since then (Kuflik et al., 1986). Although the technique has been shown to be very effective in providing analgesia and muscle relaxation around the shoulder joint, there are no published reports of its use to reduce shoulder dislocations in the accident and emergency department. At present the majority of dislocated shoulders are reduced under general anaesthesia, intravenous sedation with or without analgesia, or nitrous oxide. We undertook a prospective study using interscalene blocks to provide regional anaesthesia for the manipulation of shoulder dislocations between July and October 1988.

\section{METHODS}

All patients with a dislocation of the glenohumeral joint who attended the department during the study period were asked to participate in the study. An interscalene block,

Correspondence: T. F. Underhill, Senior Registrar, Accident and Emergency Department, Queens Medical Centre, University Hospital, Nottingham NG7 2UH, England. 
based on the technique described by Winnie but with certain modifications, was carried $\frac{\mathrm{D}}{6}$ out by one of the authors following clinical diagnosis of the injury. Five minutes after 3 the insertion of the block the patient was sent for X-ray with a nurse escort. On the? patient's return to the department following radiological confirmation of the diagnosis, $\overrightarrow{\vec{F}^{*}}$ the efficacy of the block was assessed and manipulation performed. Both subjective and objective assessment of each block was made. Patients were asked to state whether the음 pain was worse, the same, improved or absent following the block. The range of passive $\frac{\overline{\bar{\omega}}}{\overrightarrow{0}}$

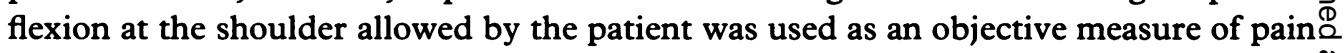
relief. The ease of reduction and the power of active abduction following relocation using MRC grading were recorded. The Hippocratic method was used most often to $\overrightarrow{0}$ effect reduction and the patient's arm then rested in a sling. All patients were reviewed $\overrightarrow{\vec{H}}$ within 2 days in the Fracture Clinic and any complications of the nerve block still $\stackrel{\omega}{\circ}$ present were noted.

\section{TECHNIQUE}

The technique used is based on that of Winnie (1970) but modified because only the $\frac{\Phi}{0}$ shoulder and not the whole arm needs to be anaesthetic. A cannula is inserted into the $\mathbb{\Phi}$ opposite arm to provide venous access if required. The patient is laid semirecumbent $\overline{0}$ and asked to turn his head away from the side of injury. The operator identifies the $\stackrel{\mathbb{D}}{\sim}$ lateral border of sternomastoid at the level of the cricoid cartilage and moves the palpating finger laterally across scalenus anterior until the next groove is felt. This is the interscalene groove. Elements of the brachial plexus may be rolled under the palpating finger in the groove and moderate pressure may elicit paraesthesia in thin patients. The external jugular vein often crosses the interscalene groove at this point and can provide a useful additional landmark.

The skin is stabilized with a finger across the groove on either side of the point of $\overrightarrow{\vec{O}}$ entry. A $25 \mathrm{~mm} 23$-gauge needle on a $20 \mathrm{ml}$ syringe is inserted perpendicular to the skin 3 in all three planes, that is medially, caudally and posteriorly. The brachial plexus sheath lies $1 \mathrm{~cm}$ to $2 \mathrm{~cm}$ deep to the skin surface at this point. Paraesthesia may be elicited but is not essential for the success of the block. Check aspiration is performed to avoid arterial $\stackrel{\infty}{3}$. puncture. Cranial spread of local anaesthetic during injection is minimized by finger pressure, and following injection the local anaesthetic is massaged caudally. A total $\stackrel{3}{-}$. volume of $30 \mathrm{mls}$ is injected and in this study patients received $20 \mathrm{mls}$ of $1 \%$ lignocaine with adrenaline 1 in 200,000 and $10 \mathrm{mls}$ of $0.5 \%$ prilocaine.

\section{RESULTS}

The age range of patients in this study was 16 to 94 with a mean age of 42 years. There were 29 patients in the study with a total of 30 dislocations of which 29 were anterior and one was posterior. One patient with a history of recurrent dislocation presented ${ }_{\bar{\Phi}}$ twice. The pain was abolished in 14 blocks and improved in a further 13 (Table 1). In $3 \stackrel{?}{+}$ 
Table 1 Change in pain following interscalene block

\begin{tabular}{lrl}
\hline Abolished & 14 & $(14$ reduced $)$ \\
Improved & 13 & $(12$ reduced $)$ \\
Unchanged & 3 & $(0$ reduced $)$ \\
Total & 30 & $(26$ reduced $)$ \\
\hline
\end{tabular}

blocks the pain remained unchanged and reduction was unsuccessful in these cases. One patient who reported an improvement in the degree of pain also had an unsuccessful manipulation. Reduction was carried out without difficulty in the 26 successful blocks. The range of passive flexion permitted by the patient following the block was greater than $45^{\circ}$ in 24 of the 26 successful manipulations (Table 2) and muscle power was reduced (MRC grade 3 or less) in 21 of the 26 reductions (Table 3). Paraesthesia was elicited in only 7 of the 26 reductions. The time between injection and onset of anaesthesia varied from 10 to $30 \mathrm{~min}$. Duration of the nerve blocks was not measured and many patients were discharged from the department before the block had completely worn off. There were no significant complications following interscalene block. Twelve patients developed a transient Horner's syndrome and two patients experienced a hoarse voice (Table 4).

Table 2 Range of passive flexion following block

\begin{tabular}{lrl}
\hline More than $45^{\circ}$ & 24 & $(24$ reduced $)$ \\
$10-45^{\circ}$ & 4 & $(2$ reduced $)$ \\
Less than $10^{\circ}$ & 2 & $(0$ reduced $)$ \\
Total & 30 & $(26$ reduced $)$ \\
\hline
\end{tabular}

Table 3 Muscle power following reduction

\begin{tabular}{llr}
\hline MRC grade & $0-1$ & 8 \\
& $2-3$ & 13 \\
& $4-5$ & 5 \\
& Total & 26 \\
\hline
\end{tabular}

Table 4 Complications - total 30 blocks

\begin{tabular}{lr}
\hline Horner's & 12 \\
Hoarseness & 2 \\
\hline
\end{tabular}




\section{DISCUSSION}

Interscalene brachial plexus block provides good pain relief and muscle relaxation for the patient with a dislocation of the shoulder. The success of manipulation in this study is closely associated with the success of the interscalene block and as with any method of regional anaesthesia there is a small but significant failure rate. Of the four failed reductions three blocks failed completely and one block produced subjective pain relief only. All four failed blocks occurred in the first 2 months of the study period. Only one of the authors had used this technique prior to the study and with greater experience we expect our success rate to match that quoted by other authors, that is $91 \%$ (Ward, 1974) to $93 \%$ (Kuflik et al., 1986). The use of a nerve stimulator would allow a greater accuracy in positioning the tip of the needle and might therefore be expected to raise the success rate still further.

In four published series of interscalene blocks (Winnie, 1970; Ward, 1974; VesterAnderson et al., 1981 and Kuflik et al., 1986), with a total of 422 patients, the only complications were phrenic nerve palsy in up to $5 \%$ of cases and recurrent laryngeal palsy in up to $17 \%$ of cases. Horner's syndrome, of which the patient is usually unaware, occurs in up to $75 \%$ of interscalene blocks. Case reports of more significant complications have included cardiac arrest (Edde \& Deutsch, 1977), spinal anaesthesia (Ross \& Scarborough, 1973), epidural anaesthesia (Scammell, 1979) and permanent nerve damage (Barutell et al., 1980). In the first and last of these reports needle lengths of $6 \mathrm{~cm}$ and $8.8 \mathrm{~cm}$ respectively were used. The nerve supply to the shoulder is carried predominantly by the roots of $\mathrm{C} 5$ and $\mathrm{C} 6$ and lies quite superficial in the interscalen 8 groove. Winnie's original description of the technique includes walking the point of the needle off the transverse process in order to achieve a complete brachial plexus block? This is not necessary if only anaesthesia of the shoulder is required and we suggest that by using a needle of not more than $3 \mathrm{~cm}$ in length and minimizing the cranial spread of local anaesthetic, the incidence of significant complications is extremely small. Bilateral blockade must be avoided because of the risk of phrenic or recurrent laryngeal nerve palsy.

In order to become accepted a technique such as this must show that it has advantages over those methods in current use. Ideally an anaesthetic technique should provide analgesia, be easy to perform, be safe and have a high success rate. If a dislocated joint is to be manipulated, muscle relaxation is also desirable. The alternative methods of anaesthesia used for shoulder manipulations include intravenous sedation with or without intravenous analgesia, general anaesthesia and nitrous oxide. Of these general anaesthesia provides the best conditions for reduction with good analgesia and muscle relaxation, but the disadvantages are the delay before the anaesthetic can be administered and the length of recovery period which may necessitate hospital admission. Intravenous sedation alone produces amnesia with little analgesia or muscle relaxation. The technique is easy to perform and although widely practised there is a significant risk of respiratory depression and complete apnoea has been reported (Smith \& Aitkenhead, 1985). Adding intravenous analgesia provides pain relief but increases the risk of respiratory depression. The incidence of failed manipulation with this method has been reported to be approximately 5\% (Royle, 1973). There is also the risk of inhalation of gastric contents and ideally the procedure should not be carried out within 
$6 \mathrm{~h}$ following a meal. Nitrous oxide is a safe but relatively weak anaesthetic agent and is combined with oxygen in equal volumes as Entonox. It provides reasonable analgesia but has no relaxant properties and is user dependent. Interscalene brachial plexus block of the shoulder provides excellent conditions for manipulation, is easy to perform and relatively quick to take effect. The disadvantages of the technique are a failure rate of approximately $10 \%$ and the potential hazards associated with incorrect needle placement.

The exact volume of local anaesthetic required to block the shoulder only depends on the size of the patient and the accuracy of needle placement. To achieve a complete brachial plexus block Winnie suggested using a volume of $0.5 \mathrm{ml}$ per inch of the patient's height and Ward (1974) used 30-35 mls of $1.5 \%$ prilocaine. If paraesthesia of the shoulder is elicited when the needle is inserted $20 \mathrm{mls}$ of local anaesthetic may be adequate, but without paraesthesia $30 \mathrm{mls}$ will probably be required. Measurement of plasma concentrations of local anaesthetics showed that even when combined with adrenaline, lignocaine produced significantly greater plasma concentrations than the same dose of plain prilocaine (Wildsmith et al., 1977). In choosing to use $20 \mathrm{mls}$ of $1 \%$ lignocaine with adrenaline and $10 \mathrm{mls}$ of $0.5 \%$ plain prilocaine we have tried to balance the advantage of lignocaine with its shorter duration of action and the disadvantage of its potential toxicity.

\section{ACKNOWLEDGEMENTS}

The authors are grateful to Dr Cartwright, Consultant Anaesthetist, Derbyshire Royal Infirmary, for his advice and encouragement and to Sue Pollicott for her help in preparing the manuscript

\section{REFERENCES}

Barutell C., Vidal F., Raich M. \& Montero A. (1980) A neurological complication following interscalene brachial plexus block. Anaesthesia 35, 365-7.

Edde R. R. \& Deutsch S. (1977) Cardiac arrest after interscalene brachial plexus block. Anaesthesia and Analgesia 56, 446.

Kuflik P., Ankolekar A., Stuchim S., et al. (1986) Interscalene brachial plexus block anaesthesia for upper extremity surgery. fournal of Hand Surgery 11, 246-8.

Ross S. \& Scarborough C. D. (1973) Total spinal anaesthesia following brachial plexus block. Anaesthesiology 39, 458.

Royle G. (1973) Treatment of acute anterior dislocation of the shoulder. British fournal of Clinical Practice 27, 403-5.

Scammell S. J. (1979) Inadvertent epidural anaesthesia as a complication of interscalene brachial plexus block. Anaesthesia and Intensive Care 7, 56-7.

Smith G. \& Aitkenhead A. R. (1985) Textbook of Anaesthesia. Churchill Livingstone, Edinburgh.

Vester-Andersen T., Christiansen C., Hansen A. et al., (1981) Interscalene brachial plexus block: area of analgesia, complications and blood concentrations of local anaesthetics. Acta Anaesthesia Scandinavia 25, $81-4$. 
$204 T . \mathcal{F}$. Underhill et al.

Ward M. E. (1974) The interscalene approach to the brachial plexus. Anaesthesia 29, 147-57.

Wildsmith J., Tucker G., Cooper S. et al., (1977) Plasma concentrations of local anaesthetics after 3 interscalene brachial plexus blocks. British fournal of Anaesthesia 49, 461-5.

Winnie A. P. (1970) Interscalene brachial plexus block. Anaesthesia and Analgesia: Current Researches 49, 455-66. 